PROCEEDINGS OF THE

AMERICAN MATHEMATICAL SOCIETY

Volume 35, Number 2, October 1972

\title{
INTEGRABLY PARALLELIZABLE MANIFOLDS
}

\author{
VAGN LUNDSGAARD HANSEN
}

\begin{abstract}
A smooth manifold $M^{n}$ is called integrably parallelizable if there exists an atlas for the smooth structure on $M^{n}$ such that all differentials in overlap between charts are equal to the identity map of the model for $M^{n}$. We show that the class of connected, integrably parallelizable, $\boldsymbol{n}$-dimensional smooth manifolds consists precisely of the open parallelizable manifolds and manifolds diffeomorphic to the $n$-torus.
\end{abstract}

1. Introduction. In this note $M^{n}$ is an $n$-dimensional, paracompact smooth manifold without boundary, and $G$ is an arbitrary subgroup of the general linear group $\mathrm{Gl}(n, \boldsymbol{R})$ on $\boldsymbol{R}^{n}$.

Definition. $M^{n}$ is called $G$-reducible if the structural group of the tangent bundle for $M^{n}$ can be reduced from $\operatorname{Gl}(n, R)$ to $G . M^{n}$ is called integrably $G$-reducible if there exists an atlas $\left\{\left(U_{i}, \theta_{i}\right)\right\}$ for the smooth structure on $M^{n}$ such that the differential in overlap between charts $\left(\theta_{i} \circ \theta_{j}^{-1}\right)_{* x}$ belongs to $G$ for all $x \in \theta_{j}\left(U_{i} \cap U_{j}\right) \subseteq R^{n}$ and all $i, j$ in the index set for the atlas.

It is clear that an integrably $G$-reducible manifold is $G$-reducible and therefore the following problem naturally arises.

Problem. Classify for a given $G$ those $G$-reducible manifolds which are integrably $G$-reducible.

Let us illustrate this problem with two examples.

EXAMPLE 1. Let $G=O(n)$ be the orthogonal group. Any manifold $M^{n}$. is $O(n)$-reducible, since it admits a Riemannian metric. On the other hand it is easy to see that $M^{n}$ is integrably $O(n)$-reducible if and only if it admits a flat Riemannian metric. (A flat Riemannian manifold is locally isometric to $\boldsymbol{R}^{n}$.)

EXAmPle 2. Suppose $n=2 k$ and let $\mathrm{Gl}(k, \boldsymbol{C})$ be the general linear group on $\boldsymbol{C}^{k}$ considered as a subgroup of $\mathrm{Gl}(n, \boldsymbol{R})$ under the usual identification of $\boldsymbol{C}^{k}$ with $\boldsymbol{R}^{n}$. Then $\boldsymbol{M}^{n}$ is $\operatorname{Gl}(k, C)$-reducible if and only if it admits an almost complex structure and integrably $\operatorname{Gl}(k, C)$-reducible if and only if it admits a complex structure. The classification of the manifolds admitting a complex structure among those admitting an almost complex structure is far from being complete.

Received by the editors November 8, 1971 and, in revised form, February 28, 1972. AMS 1970 subject classifications. Primary 53C10, 57D30.

Key words and phrases. Tangent bundle, reduction of structural group, integrable reduction, parallelizable manifold, rank of a manifold.

(. American Mathematical Society 1972 
For open manifolds Haefliger has recently given a nice reformulation of the problem using the work on foliations of Phillips and Gromov. See e.g. Haefliger [3, Example 2]. Notice here that an integrably $G$-reducible manifold in our sense in Haefliger's terminology is a manifold which admits a $G$-structure. For closed manifolds very little seems to be known.

The purpose of this note is to give the complete solution to the problem in the case where $G=G_{0}$ is the identity subgroup of $\mathrm{Gl}(n, R)$. It is easy to see that a manifold $M^{n}$ is $G_{0}$-reducible if and only if it is parallelizable. Therefore we will call an integrably $G_{0}$-reducible manifold integrably parallelizable. With this notation we have

THEOREM. Let $M^{n}$ be connected and parallelizable. Then $M^{n}$ is integrably parallelizable in precisely the following cases:

(i) $M^{n}$ is open;

(ii) $M^{n}$ is diffeomorphic to the $n$-dimensional torus $T^{n}=S^{1} \times \cdots \times S^{1}$.

This theorem answers a question of J. Eells, who asked for a determination of the finite dimensional integrably parallelizable smooth manifolds after the discovery that any smooth separable Hilbert manifold is integrably parallelizable in a very strong sense (see the remark in §3).

2. Proof of the theorem. For the proof of the theorem we need two well-known results which we state as lemmas. First a lemma of Frobenius type (see e.g. Hicks [4, p. 128]).

LEMMA 1. Let $M^{n}$ be a parallelizable smooth manifold parallelized by the smooth vector fields, $X_{1}, \cdots, X_{n}$. Suppose that these vector fields commute, i.e. all Lie brackets $\left[X_{i}, X_{j}\right]=0$. Then each point $x \in M^{n}$ has a coordinate neighbourhood $(U, \theta)$ such that the restrictions of the vector fields $X_{1}, \cdots, X_{n}$ to $U$ coincide with the coordinate vector fields on $U$.

Recall now that the rank of a smooth manifold $M^{n}$ is the maximal number of linearly independent, commuting smooth vector fields which can be defined on the manifold. Then we have

LEMMA 2. A compact, connected smooth manifold $M^{n}$ has rank $n$ if and only if it is diffeomorphic to the $n$-torus $T^{n}=S^{1} \times \cdots \times S^{1}$.

Proof. This result was originally obtained by Willmore [8, Theorem 2]. We offer here an alternative proof. Assume that $M^{n}$ has rank $n$. There is then an action of $\boldsymbol{R}^{n}$ (considered with its standard abelian Lie group structure) on $M^{n}$ such that all the orbits for the action are immersed submanifolds; see e.g. Rosenberg [7]. For an arbitrary point $x \in M^{n}$ consider now the isotropy group $\boldsymbol{R}_{x}^{n}$ for such an action at $x$ and its quotient group $G_{x}=\boldsymbol{R}^{n} / \boldsymbol{R}_{x}^{n}$ in $\boldsymbol{R}^{n}$. The orbit map $o_{x}: \boldsymbol{R}^{n} \rightarrow M^{n}$ at $x$ induces then a map 
$\bar{o}_{x}: G_{x} \rightarrow M^{n}$. Since $\boldsymbol{R}_{x}^{n}$ is a closed discrete subgroup of $\boldsymbol{R}^{n}, G_{x}$ can be given the structure of an abelian Lie group such that the canonical projection $\boldsymbol{R}^{n} \rightarrow G_{x}$ is a Lie group homomorphism. It is easy to prove that $o_{x}$ is surjective and then it follows immediately that $\bar{o}_{x}$ is a diffeomorphism. But then $G_{x}$ is a compact, connected abelian Lie group and hence diffeomorphic to $T^{n}$. Thus $M^{n}$ is also diffeomorphic to $T^{n}$. Since conversely $T^{n}$ clearly has rank $n$ the proof is finished.

PROOF OF THE THEOREM. (i) Suppose first that $M^{n}$ is open. By a result of Hirsch [5, Theorem 4.7] there exists then an immersion $F: M^{n} \rightarrow \boldsymbol{R}^{n}$. Locally this immersion is a diffeomorphism and we can therefore define an atlas $\left\{\left(U_{i}, \theta_{i}\right)\right\}$ on $M^{n}$, such that $\theta_{i}=F \mid U_{i}$. But then it is clear that all differentials in overlap between charts $\left(\theta_{i} \circ \theta_{j}^{-1}\right)_{* x}=1_{R^{n}}$, the identity on $\boldsymbol{R}^{n}$. Hence $M^{n}$ is integrably parallelizable.

(ii) Suppose now that $M^{n}$ is compact. If we can show that $M^{n}$ is integrably parallelizable if and only if it has rank $n$, then Lemma 2 will finish the proof of the theorem. Suppose therefore first that $M^{n}$ has rank $n$ and choose $n$ linearly independent, commuting smooth vector fields $X_{1}, \cdots, X_{n}$ on $M^{n}$. By Lemma 1 we can define an atlas $\left\{\left(U_{i}, \theta_{i}\right)\right\}$ on $M^{n}$ such that the coordinate vector fields on $U_{i}$ coincide with the restrictions of the vector fields $X_{1}, \cdots, X_{n}$ to $U_{i}$. But then it is easy to see that all differentials $\left(\theta_{i} \circ \theta_{j}^{-1}\right)_{* x}=1_{R^{n}}$ and hence $M^{n}$ is integrably parallelizable. Suppose next that $M^{n}$ is integrably parallelizable and let $\left\{\left(U_{i}, \theta_{i}\right)\right\}$ be an atlas on $M^{n}$ with all differentials $\left(\theta_{i} \circ \theta_{j}^{-1}\right)_{* x}^{-1}=1_{R^{n}}$. It is then easy to see that there exist $n$ well-defined smooth vector fields $X_{1}, \cdots, X_{n}$ on $M^{n}$, whose restrictions to $U_{i}$ coincide with the coordinate vector fields on $U_{i}$ for any chart $\left(U_{i}, \theta_{i}\right)$ in the atlas. Since coordinate vector fields commute it is clear that $X_{1}, \cdots, X_{n}$ are $n$ linearly independent, commuting smooth vector fields on $M^{n}$. Hence $M^{n}$ has rank $n$. As already observed this finishes the proof.

3. Examples and a remark. In this section we give first some examples of integrably parallelizable manifolds.

EXAMPLE 3. Every open Lie group or punctured compact, connected Lie group is integrably parallelizable.

EXAMPLE 4. Every orientable 3-manifold is parallelizable. Hence the class of connected integrably parallelizable 3-manifolds consists of all open connected orientable 3-manifolds plus diffeomorphic images of the 3-torus.

EXAmPle 5. Let $V_{n, k}$ be the Stiefel manifold of orthonormal $k$-frames in $\boldsymbol{R}^{n}$. By a theorem of Borel and Hirzebruch [1] this is always a $\pi$-manifold (stably trivial tangent bundle). Since an open $\pi$-manifold is parallelizable (an open manifold $M^{n}$ has a complex of dimension $n-1$ as 
deformation retract) it follows that all punctured Stiefel manifolds are integrably parallelizable.

EXAMPLE 6. Let $M^{n}$ be any compact, connected $\pi$-manifold. Then $M^{n} \times S^{1}$ is parallelizable but not integrably parallelizable unless $M^{n}$ is diffeomorphic to $T^{n} . M^{n} \times \boldsymbol{R}^{1}$ is also parallelizable but as an open manifold now even integrably parallelizable.

We finish with a remark concerning infinite dimensional manifolds.

REMARK. Let $X$ be an infinite dimensional separable smooth manifold modelled on the separable Hilbert space $E$. By a theorem of Kuiper [6] the general linear group $\mathrm{Gl}(\boldsymbol{E})$ on $E$ is contractible. This implies that $X$ is parallelizable. By the recent theorem of Eells and Elworthy [2] $X$ is diffeomorphic to an open subset of $E$. The parallelization of $X$ can therefore be realized by a single coordinate chart. This implies of course that $X$ is integrably parallelizable in a very strong sense. In finite dimensions it would have been too much to ask for realization of a parallelization by a single coordinate chart. The punctured 2-torus e.g. is integrably parallelizable in our sense but is not diffeomorphic to an open subset of $\boldsymbol{R}^{2}$.

\section{REFERENCES}

1. A. Borel and F. Hirzebruch, Characteristics classes and homogeneous spaces. III, Amer. J. Math. 82 (1960), 491-504. MR 22 \#11413.

2. J. Eells and K. D. Elworthy, Open embeddings of certain Banach manifolds, Ann. of Math. (2) 91 (1970), 465-485. MR 41 \#7725.

3. A. Haefliger, Lectures on the theorem of Gromov, Proc. Liverpool Singularities Sympos. II, Lecture Notes in Math., no. 209, Springer-Verlag, Berlin and New York, 1971, pp. 128-141.

4. N. J. Hicks, Notes on differential geometry, Van Nostrand Math. Studies, no. 3, Van Nostrand, Princeton, N.J., 1965, MR 31 \#3936.

5. M. W. Hirsch, On imbedding differentiable manifolds in euclidean space, Ann. of Math. (2) 73 (1961), 566--571. MR 23 \#A2223.

6. N. H. Kuiper, The homotopy type of the unitary group of Hilbert space, Topology 3 (1965), 19-30. MR 31 \#4034.

7. H. Rosenberg, Actions of $R^{n}$ on manifolds, Comment. Math. Helv. 41 (1966/67), 170-178. MR 34 \#6794.

8. T. J. Willmore, Connections for systems of parallel distributions, Quart. J. Math. Oxford Ser. (2) 7 (1956), 269-276. MR 20 \#4299.

Mathematics Institute, University of Warwick, Coventry, England

Mathematics Institute, University of Aarhus, Aarhus, Denmark

Current address: Mathematics Institute, University of Copenhagen, Copenhagen, Denmark 